\title{
DESCRIZIONE MORFOLCGICA E STIMA DELL'IMPATTO AMBIENTALE DERIVANTE DALL'APERTURA AL PUBBLICO DELLE GROTTE DI «IS ZUDDAS» PRESSO SANTADI (Nuoro, Sardegna, Italia)
}

\author{
Roberto Curreli* , Nicolino Diana** , Antonello Floris ${ }^{* * *}$, Antonello Sulas ${ }^{+}$
}

\author{
SUMMARY \\ [Morphological description and environmental impact evaluation due to the \\ development as a show cave of the "Grotte di Is Zuddas" (Nuoro, Sardinia, Italy]
}

After a short history of the discovery of the caves of "Is Zuddas" a geological description of the region and the geomorphological evolution of the cave are recorded. The results of a preliminary climatological research are reported and a rough evaluation of the visitors capacity is also given.

\section{RIASSUNTO}

Dopo una breve storia delle grotte di "Is Zuddas" a partire dalla loro scoperta, viene riportata una descrizione della geologia della zona insieme all'evoluzione geomorfologica della cavita. Sono infine forniti $i$ risultati preliminari di uno studio climatologico nonché una valutazione approssimata della capacità ricettiva della grotta.

\section{PREMESSA}

Le grotte sono degli ambienti naturali molto delicati, creatisi in centinaia di migliaia di anni ed, al contrario di altri ambienti o situazioni, una volta alterato il proprio interno, tale alterazione diventa praticamente irreversibile. Il rischio è appunto quello di deteriorare irrimediabilmente l'ipogeo se un qualsiasi sconsiderato intervento umano non tiene presente alcuni fondamentali fattori.

Per quanto riguarda le grotte disseminate in Sardegna (circa 1600), la mancanza di una legge di tutela, sempre in riferimento alla Sardegna, espone le cavità naturali ad una serie di pericoli causati da fenomeni di inciviltà: taglio di concrezioni che hanno impiegato migliaia di anni a formarsi, le batterie abbandonate, il carburo lasciato a terra, la caccia a reperti biologici, sono tra $\mathrm{i}$ più comuni.

Nelle grotte turistiche, paradossalmente il discorso varia rispetto al caso precedente, per i seguenti motivi:

a) Vengono turisticizzate soltanto le grotte cne, dal punto di vista estetico, siano interessanti e quindi precedentemente difese dall'assalto vandalico.

\footnotetext{
* Via S. Pietro 61, I-09010 NUXIS (Cagliari) Italia

** Via Carbonia, I-09010 SANTADI (Cagliari) Italia

*** Cas. Post. 17, I-09126 CAGLIARI, Italia

+ Via Giardini 11, I-09010 SANTADI (Cagliari) Italia
} 
b) Si ha tutto l'interesse a mantenere intatto nel tempo l'ambiente, in quanto fonte di reddito.

La Grotta Is Zuddas per quanto riguarda il punto a) è certamente all'avanguardia nell'isola poichè, prima i soci dello Speleo Club Sandadese e poi quelli della Cooperativa Monte Meana, hanno chiuso la grotta per proteggerla dai vandali, dormivano a turno nelle sue vicinanze tutte le notti di tutto l'anno per sorvegliarla, finché è stato ottenuto il vincolo paesaggistico ministeriale sulla stessa. Oggi, la sua incomparabile bellezza è debitrice proprio a quest'opera di costante salvaguardia.

Per il punto b) occorre tenere sotto controllo alcuni aspetti tra i quali il più importante riguarda un corretto rapporto tra visitatori ed ambiente: occorre cioè tenere sotto controllo gli effetti dell'impatto antropico che non deve mai creare delle situ azioni di irreversibilità ma essere armonico.

L'impatto antropico, se non tenuto in debita considerazione, potrebbe provocare l'innalzamento della temperatura ed una maggiore concentrazione di anidride carbonica, fattori apparentemente innocui ma di straordinaria importanza. E' noto che quando, tra una visita e l'altra, oppure tra un giorno e l'altro, nell'ambiente ipogeo i valori ambientali tornano normali l'impatto antropico non produce effetti negativi. Quando invece la variazione diventa irreversibile le conseguenze sull'ambiente possono risultare molto gravi.

Il monitoraggio continuo dei parametri ambientali ha il compito di consentire l'apporto di eventuali correttivi secondo i risultati ottenuti, preservando nel tempo un bene di inestimabile valore e fonte di reddito.

In Sardegna non esistono simili reti di monitoraggio nelle grotte turistiche ed anche in Italia sono ancora pochi, anche se vi è la tendenza ad aumentarne la diffusione.

\section{INQUADRAMENTO GEOLOGICO-STRUTTURALE}

Il settore interessato comprende terreni che vanno dal Paleozoico (Auct.) sino al Quaternario, ad esclusione del Mesozoico.

Al Paleozoico appartengono la serie Cambro-Ordoviciana. Procedendo dal basso verso l'alto si trova la Formazione di Nebida (Cambrico inf.) suddivisa in due membri: meno fini.

1) Membro di Matopa costituito da alternanze di metarenarie più o

2) Membro di Punta Manna caratterizzato dalla presenza di abbondanti lenti carbonatiche (calcari ad Archeociatine).

Subito dopo si trova la Formazione di Gonnesa (Cambrico inf.). In questa formazione puramente carbonatica, con una potenza è di circa 800 metri, si apre la cavità carsica oggetto di studio. Al suo interno si possono distinguere tre membri:

1) Membro della Dolomia rigata.

2) Membro della Dolomia grigia massiva.

3) Membro del Calcare ceroide.

Il primo è costituito da alternanze di lamine dolomitiche chiare e scure, tali rocce sono scarsamente carsificabili. La seconda unità, nella parte alta 
risulta in eteropia di facies con il calcare ceroide, queste sono costituite da Dolospariti equigranulari, definite da rotture non regolari con successiva dolomitizzazione diagenetica molto spinta. Pare si sia formata per processi di metasomatosi con il calcare ceroide, ovvero deriverebbe da quest'ultimo per vicarianza fra ioni $\mathrm{Ca}++\mathrm{e}$ ioni $\mathrm{Mg}++$.

Il Membro del calcare ceroide rappresenta la parte alta della Formazione di Gonnesa, e presenta vari litotipi. Sono stati riscontrati nel settore il calcare nero, il calcare bianco, ed il calcare grigio «ceroide». La loro rottura è concoide e presentano un'alta percentuale di carsificazione, al contrario delle dolomie grigie. Il colore del calcare nero pare sia dato dalla presenza di sostanze carboniose. Esistono anche facies ricristallizzate sicuramente prodotte dalla vicinanza del batolite granitico del complesso Monte Lattias-Pantaleo.

Il calcare grigio invece è una microsparite molto scheggiosa e così anche il calcare bianco. Successivamente affiora la Formazione di Cabitza (Cambrico medio-Ordoviciano inf.) la cui base è costituita dal alternanze di letti calcarei con letti terrigeni (siltiti ed argilliti). Il tetto, invece, è puramente detrico (argilloscisti).

Questa formazione, nella quale sono assenti fenomeni carsici, è costituita da due membri:

1) Membro dei Calcari modulari

2) Membro degli argilloscisti.

Successivamente si passa al Cenozoico con una formazione continentale, la Formazione del Cixerri (Eocene inf. - Ologocene sup.) con alternanze di arenarie silt ed argille di colore che varia dal grigio al rosso al violetto. In essa si notano spesso dei livelli calcari di scarsa potenza di origine puramente lacustre. Esiste, sempre dell' Oligocene, il complesso vulcanico di Pani LorigaPranedda. Alla base si trovano andesiti a pirosseno, nella parte intermedia si trovano dei tufi pomicei dacitici o riodacitici.

La parte alta presenta delle piroclastiti in facies ignimbritica costituite prevalentemente da rioliti e riodaciti.

Il quaternario è costituito da alluvioni antiche e alluvioni recenti separate da livelli travertinosi formatisi prevalentemente dalla vicinanza di sorgenti carsiche.

L'assetto tettonico particolarmente complicato del blocco sulcitano crea anche in quest'area complicazioni di interpretazione dei dati. E' valido il modello proposto da Arthaud (1970), Carmignani et alii (1982). Essi distinguono quattro fasi plicative con direzioni diverse:

1) Fase Sarda: ha dato blandi piegamenti con assi E-W.

2) Prima fase ercinica: blande pieghe E-W che accentuano le precedenti.

3) Seconda fase ercinica: pieghe dirette N-S.

4) Terza fase ercinica: modeste pieghe in varie direzioni.

Successivamente alla tettonica ercinica si ha la tettonica alpina che, al contrario della precedente, di tipo compressivo, questa è di tipo distensivo, ed ha formato delle strutture a Horst-Graben.

La valle di Is Zuddas potrebbe essere interpretata come una serie di faglie parallele orientate NE-SW le cui linee tettoniche principali hanno creato una zona di debolezza in cui si è impostata la grotta di Is Zuddas. 


\section{GEOMORFOLOGIA DELLA GROTTA}

La cavità in esame si apre nel complesso carbonatico paleozoico della «Formazione di Gonnesa» (Cambrico inf.) in località Is Zuddas, comune di

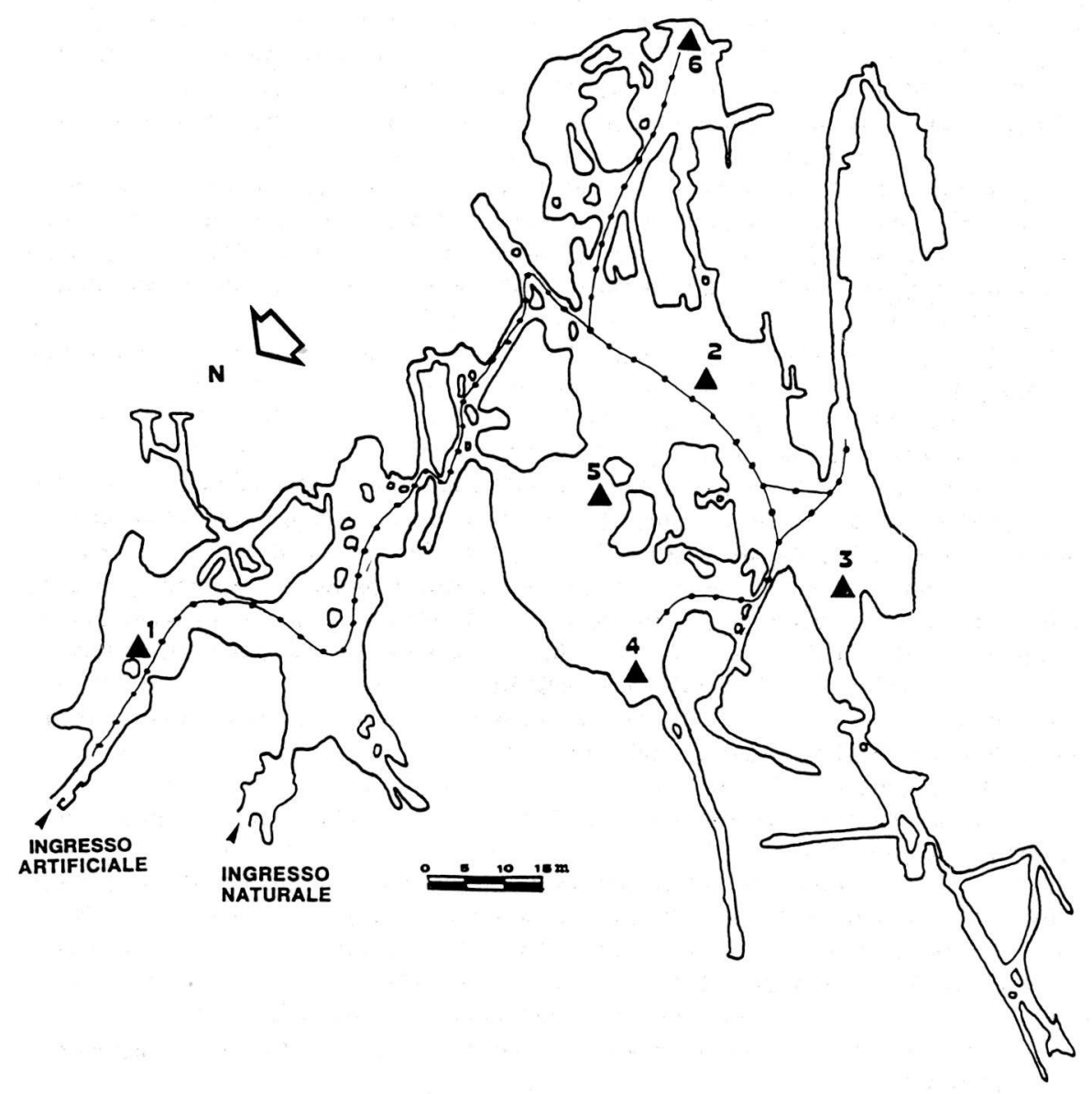

Fig. 1 - Pianta della Grotta di Is Zuddas. Sono anche indicati: il percorso turistico e le stazioni di monitoraggio: 1 - La Colata; 2 - L'Organo; 3 - L'Organo (zona delle aragoniti); 4 - Sala del Teatro (a circa $15 \mathrm{~cm}$ da un faro); 5 - Sala del Teatro (zona alta); 6 - Sala delle Eccentriche. 
Santadi, Provincia di Cagliari, individuata nella Carta d'Italia 1:25.000 dell'Istituto Geografico Militare (foglio 233 II SO), lungo una serie di faglie parallele che hanno determinato l'approfondirsi dell'alveo del Rio Cambudu, colmato in periodi recenti, da alluvioni quaternarie.

L'impostazione della grotta segue l'evoluzione di un reticolo di fratture perpendicolari tra loro orientate principalmente $\mathrm{N} 70^{\circ}$ e N $340^{\circ}$. All'incrocio di queste fratture si sono formati degli ambienti di considerevoli dimensioni, come la Sala del crollo (m 40 x 30). In quest'ultima sono ben visibili dei canali di volta che seguono le linee tettoniche precedentemente citate.

L'evoluzione di queste sale non è avvenuta soltanto per fenomeni chimici, ma anche per fenomeni meccanici, soprattutto a seguito di cedimenti di parti della volta che hanno prodotto massi di enormi dimensioni. Nella Sala del crollo, ma anche in altre parti della grotta, si notano fratture beanti di circa 3 centimetri, e dei ribaltamenti di blocchi con un rovesciamento obliquo di circa 50 gradi. Tutto ciò è stato probabilmente prodotto da erosione al di sotto del pavimento per opera delle acque freatiche.

L'accesso alla cavità si presenta come un tunnel artificiale utilizzato negli anni sessanta come cava di alabastro. L'ingresso naturale si trova a circa 30 metri a W e ad una trentina di metri più in alto come quota. Come forme di erosione si notano delle condotte forzate di forma circolare od ellitica. In alcune sale della cavità, come la Sala delle eccentriche, è manifesta l'evoluzione dei vani fusiformi, poi riempiti da sedimenti carbonatici. Oltre a delle vaschette superficiali alimentate da stillicidio, dal punto di vista idrogeologico esiste un lago sifone che evidenzia il livello della falda acquifera a circa 115 metri di quota (Fig. 1).

Per quanto attiene la sedimentologia, si possono classificare il deposito: in due tipi: detritici e carbonatici.

I primi possono essere ancora suddivisi in due, sottotipi: da crolli di volta e da depositi da parte delle acque circolanti. Essi hanno dimensioni eterogenee, sono privi di indice di arrotondamento e presentano spigoli vivi. Tendono a riempire quasi tutte le grosse sale, spesso evolvendo in vere e proprie frane di crollo.

I secondi sono invece ben classati e si possono rinvenire in quasi tutta la cavità; principalmente sono argille e silt a giacitura orizzontale tra loro alternati, tra questi spesso sono intercalati livelli di sabbie fini. Un attento studio palinologico potrebbe determinare anche l'età di questi riempimenti.

Sempre trasportati dalle acque circolanti si possono rinvenire delle brecce ossifere, principalmente costituite da ossa di uccelli e piccoli roditori (Prolagus sardus). I riempimenti carbonatici sono numerosissimi ed hanno svariate forme. Possono distinguersi in due tipi: concrezioni semplici e cristallizzazioni.

1) Le concrezioni semplici

Queste possono essere classificate a loro volta in:

A1 - Depositi pendenti: tra essi troviamo stalattiti, stalagmiti, cannule e cortine. 
A2 - Depositi conseguenti a caduta libera dell'acqua:

tra essi si rinvengono concrezioni a splash e stalagmiti. Dal congiungimento tra le stalattiti e stalagmiti si sono formate talvolta grosse colonne.

B - Depositi dovuti a scorrimento dell'acqua lungo una superficie: tra essi si annoverano colate, crostoni, e barriere di vaschette.

C - Depositi dovuti a precipitazione di calcare di acque tranquille: si osservano tracce di livello e calcite galleggiante.

D - Depositi dovuti a moto turbolento dell'acqua: in questo caso si rinvengono perle di grotta o pisoliti.

E - Depositi dovuti a capillarità:

foglie ma soprattutto le eccentriche. Queste, con le aragoniti aciculari, sono una delle caratteristiche fondamentali della grotta. Nella parte terminale della stessa abbondano, sia come quantità che come forma. La loro genesi para sia avvenuta grazie ad una scarsa portata d'acqua nel canale interno della concrezione (frazioni di $\mathrm{cm} 3$ al giorno), variazione di pressione idrostatica e presenza di impurezze nel microcanale che, per fenomeni di tensione superficiale, riescono a far deviare le micro-gocce non rispettando la gravità.

F - «Latte di monte»:

da uno studio spettrografico il «latte di monte» di Is Zuddas non è costituito da una calcite, ma da huntite $\mathrm{CaMg} 3(\mathrm{CO} 3) 4$.

$\mathrm{G}$ - Concrezioni gluteiformi formatesi in condizioni freatiche.

\section{2) Cristallizzazioni}

Di queste si citano le aragoniti aciculari perchè insieme alle eccentriche costituiscono il maggiore interesse e richiamo della grotta. Si presentano come dei grossi ciuffi di cristalli simili a tanti aghi. Il sistema di cristallizzazione è quello rombico. La loro genesi è ancora incerta: si pensa ad una vicarianza tra ioni $\mathrm{Ca}++\mathrm{e}$ ioni $\mathrm{Mg}++$, questi ultimi con raggio ionico minore, oppure vicarianza tra ioni $\mathrm{Ca}++\mathrm{e}$ ioni $\mathrm{Pb}++, \mathrm{Ba}++, \mathrm{Zn}++, \mathrm{Fe}++, \mathrm{Sr}++$ ed altri.

\section{LE VARIAZIONI CLIMATICHE}

Nei mesi di luglio ed agosto 1990, la grotta è stata sottoposta ad un primo controllo climatico per notare eventuali variazioni climatiche da porre in relazione al tipo di flusso turistico più o meno intenso. 
Sono state individuate 6 zone caratteristiche interessate dal passaggio di visitatori che qui si elencano:

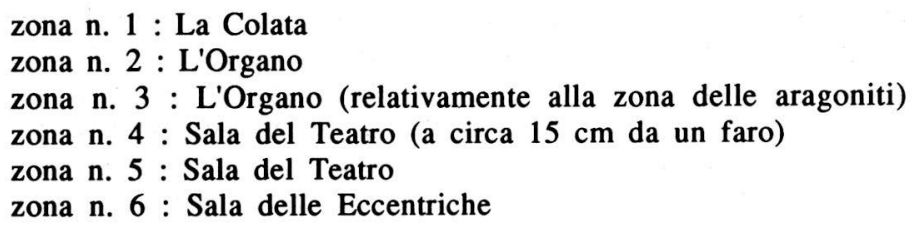

I rilevamenti sono stati effettuati in giornate con flussi diversi di visitatori, corrispondenti, rispettivamente, a 20, 240 e 1000 turisti/giorno. Il valore "naturale" della temperatura è stato rilevato in periodi di nessun flusso turistico. I rilevamenti sono stati ripetuti all'apertura antimeridiana, alla chiusura antimeridiana, all'apertura pomeridiana ed alla chiusura pomeridiana.

Nella tabella seguente sono riportati i valori così òttenuti:

\begin{tabular}{|c|c|c|c|c|c|}
\hline \multicolumn{2}{|c|}{ Turisti/giorno: } & \multirow{2}{*}{0} & \multirow{2}{*}{${ }^{20}$} & \multirow{2}{*}{$\frac{240}{{ }^{\circ} \mathrm{C}}$} & \multirow{2}{*}{${ }^{\circ} \mathrm{C}$} \\
\hline & Zona & & & & \\
\hline 1 & La Colata & 15,0 & 15,0 & 15,0 & 15,0 \\
\hline 2 & L'Organo & 15,0 & 15,0 & 15,0 & 15,0 \\
\hline 3 & L'Organo & & & & \\
\hline & (zona delle aragoniti) & 15,9 & 15,9 & 15,9 & 16,0 \\
\hline 4 & Sala del Teatro & & & & \\
\hline 5 & Sala del Teatro & $\begin{array}{l}15,9 \\
15,7\end{array}$ & $\begin{array}{l}15,9 \\
15,7\end{array}$ & $\begin{array}{l}15,9 \\
15,7\end{array}$ & $\begin{array}{l}10,4 \\
15,7\end{array}$ \\
\hline 6 & Sala delle Eccentriche & 15,7 & 15,7 & 15,8 & 15,9 \\
\hline
\end{tabular}

Nelle zone 1 e 2 la temperatura non varia passando da 0 a 20 turisti/giorno. Questo fatto è da mettere in relazione all'ampiezza degli ambienti ed al fatto che sia l'ingresso artificiale sia quello naturale (che influiscono sulla zona 1) svolgono una funzione compensativa. In particolare l'ingresso naturale si trova in una zona alta rispetto al passaggio dei turisti, per cui eventuali aumenti di temperatura vengono smaltiti facilmente. L'umidità relativa si attesta sul $95 \%$ costante nel tempo.

Con un flusso di 1000 turisti/giorno, la zona 3 ha un aumento di $0,1^{\circ} \mathrm{C}$, passando da 15,9 a $16^{\circ} \mathrm{C}$ che rimangono costanti nel tempo anche nel periodo di chiusura antimeridiana (60 minuti), per tornare al valore normali il giorno dopo, all'apertura antimeridiana.

Sempre con un flusso di 1000 turisti/giorno, nella zona 4 si registra una variazione di $0,5^{\circ} \mathrm{C}$ passando da 15,9 a $16,4^{\circ} \mathrm{C}$. Ciò però è dovuto ad un effetto diretto dei turisti ma al riscalmanto prodotto dal faro che rimane acceso per un tempo più lungo rispetto agli altri casi, dato il flusso praticamente continuo dei visitatori. 
Ciò è del resto confermato dall'invarianza della temperatura nella zona 5 , indipendentemente dall'intensità del flusso dei visitatori..

Nella zona 6 con un flusso di 1000 turisti/giorno, si registra un aumento della temperatura di $0,2^{\circ} \mathrm{C}$ alla chiusura antimeridiana, valore che scende di un decimo alla apertura pomeridiana, per risalire di un decimo alla chiusura, per cui può notarsi un aumento di $0,2^{\circ} \mathrm{C}$ alla fine di ogni turno, sia esso antimeridiano che pomeridiano. Nel giorno successivo si ritorna al valore naturale.

Sempre nella zona 6 ma con un flusso di 240 turisti/giorno si osserva un'aumento della temperatura di $0,1^{\circ} \mathrm{C}$.

Mentre nella zona 4 l'equilibrio termico tende a ristabilirsi durante la chiusura antimeridiana, nella zona 6 è necessario attendere tutta la notte per raggiungere lo stesso risultato. Ciò è dovuto sia all'ambiente alquanto ristretto, sia alla sosta di più lunga durata rispetto alle altre probabilmente forse perchè si tratta dell'angolo più suggestivo di tutta la grotta.

\section{LA RICETTIVITA'}

Per motivi strettamente economici non è stato possibile installare una rete di rilevamento che registri la temperatura e l'umidità dell'aria nonché l'intensità ed il verso delle correnti d'aria.

Comunque i primi dati, peraltro parziali e riferiti ad un breve periodo di rilevamento, mostrano che la grotta che registra punte massime intorno ai 1000 visitatori giornalieri, nel complesso, è in grado di assorbire senza particolari problemi un impatto antropico di questa intensità, riuscendo a ritornare alla situazione iniziale nell'arco di una notte, se non addirittura prima.

Rimane un dubbio circa la zona 6 che in effetti, anche per la straordinaria e concentrata presenza di concrezioni, risulta essere uno dei punti da tenere in maggiore considerazione, in una politica di salvaguardia ambientale. Un correttivo potrebbe essere dato, per esempio, da una sosta leggermente più breve dei visitatori. Inoltre le luci potrebbero essere meno intense per evitare un'altra fonte di calore. Anche una riduzione generalizzata del periodo di accensione dei fari, in modo da limitarne il funzionamento al tempo strettamente necessario contribuirebbe a ridurre l'inquinamento termico insieme all'adozione di lampade per quanto possibile "fredde"

Per il futuro potrebbe valer la pena di concentrare in questa zona le ricerche climatologiche, per valutare meglio l'effettiva ricettività ambientale. Il resto della grotta non presenta particolari problemi, anzi sembra esserci una buona sintonia tra esigenze turistiche e conservazione dell'ambiente. L'impianto di monitoraggio, attualmente allo studio, contribuirà a muoversi in questa direzione. 


\section{BIBLIOGRAFIA}

Arthaud F., 1963 - Un exemple de tectoniques exposées dans le Paléozoique de l'Iglesiente (Sardaigne). C.R. Somm. Soc. Geol. Fr., 9: 303-304.

Cabrol P., Coudray J., Dandurand J.L., Schott J., 1978 - Sur la possibilité de transformation naturelle calcite-aragonite dans les conditions ordinaires de température et pression; reproduction expérimentale du phénomène. C. R. Acad. Sc. Paris, 287, s. D: 411-414.

Carmignani L., Cocozza T., Gandin A., Pertusati P.C. (1982) - Lineamenti della geologia del'Iglesiente. Sulcis. Guida alla geologia del Paleozoico Sardo. Guide geologiche regionali. Soc. Geol. It.: 55/57.

Cervellati R., Forti P., Zavatti R., 1971 - L'aragonite azzurra sarda. Speleologia Emiliana, s. 2, 3(7): 43/60.

Cigna A.A., Forti P., 1989 - I principali agenti di degrado nelle grotte turistiche: loro effetti e possibili rimedi. In: Soc. Spel. It. e Club Alp. It. Problemi di inquinamento e salvaguardia delle aree carsiche. Nuova Editrice Apulia, Martina Franca: 47/60.

Cigna A.A., 1989 - La capacita ricettiva delle grotte turistiche quale parametro per la salvaguardia dell'ambiente sotterraneo. Il caso delle grotte di Castellana. Atti XV Congr. Naz. Speleol., Gruppo Puglia Grotte Amm. Com. Castellana Grotte: 999/1012.

Curreli R., 1989 - Rilevamento geologico $e$ inventario delle risorse geominerarie, pedologiche e fitogeografiche di una porzione del territorio di Nuxis (Sardegna meridionale). Tesi di laurea inedita: Università di Cagliari, Dip. Scienze della Terra.

Curreli R., 1988 - Speleogenesi; rapporti fra strutture geologiche e carsismo ipogeo in Sardegna. Speleologia Sarda 67: 1/12.

Di Stefano M., 1984 - Sulla Grotta Is Zuddas. Relazione inedita per "Italia Nostra".

Pensabene G., 1986 - Stucio sulle aragoniti della Risalita dei Lucchesi nell'Antro del Corchia (120. T/LU). Rivista Speleologica Toscana, 2: 2946.

Società Speleologica Italiana, 1978 - Manuale di speleologia. Longanesi, Milano.

Todde F., Barbata P., 1972 - Grotta di «Is Zuddas». Speleologia Sarda, 1(1): 510 . 